\title{
The Geminga pulsar wind nebula in the mid-infrared and submillimetre
}

\author{
J. S. Greaves ${ }^{1 \star}$ and W. S. Holland ${ }^{2}$ \\ ${ }^{1}$ School of Physics and Astronomy, Cardiff University, 4 The Parade, Cardiff CF24 3AA, UK \\ ${ }^{2}$ UK Astronomy Technology Centre, Royal Observatory, Blackford Hill, Edinburgh EH9 3HJ, UK
}

Accepted 2017 June 13. Received 2017 June 9; in original form 2017 April 19

\begin{abstract}
The nearby middle-aged Geminga pulsar has crossed the Galactic plane within the last $\sim 0.1$ Myr. We present archival data from Wide-field Infrared Survey Explorer and from SCUBA and SCUBA-2 on the James Clerk Maxwell Telescope to assess whether any midinfrared and submillimetre emission arises from interaction of the pulsar wind nebula with the interstellar medium. A candidate shell and bow shock are reported. Given the low pulsar velocity and local density, dust grains appear able to penetrate into the nebula. A compact source seen towards the pulsar is fitted with a dust spectrum. If confirmed as a real association at higher resolution, this could be a circum-pulsar disc of at least a few Earth-masses, in which future planets could form.
\end{abstract}

Key words: protoplanetary discs - ISM: bubbles - submillimetre: planetary systems.

\section{INTRODUCTION}

Geminga is a radio-quiet pulsar discovered through gamma-ray observations, with a period of $237 \mathrm{~ms}$ and a spin-down age of $0.34 \mathrm{Myr}$. The pulsation behaviour has been studied mainly at high energies, with a faint optical pulsed counterpart (Shearer et al. 1998), but only weak radio continuum, likely trailing the pulsar motion (Pellizzoni et al. 2011). Faherty, Walter \& Anderson (2007) showed that Geminga is relatively nearby, at $250(-62,+120) \mathrm{pc}$, and has a

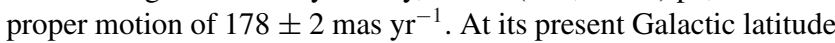
of $b=+4.3$, a plane crossing is inferred about $0.1 \mathrm{Myr}$ ago.

Sweeping of the interstellar medium (ISM) could thus shape the pulsar wind nebula $(\mathrm{PWN})$ around Geminga, rather than interaction with the supernova remnant (SNR) that the pulsar has left behind. The morphology of the PWN is unusual, as there is no obvious bowshock at the front of the nebula, although there is a candidate shock around the pulsar in the optical (Shibanov et al. 2006). The nebula has been extensively mapped in X-rays, and Posselt et al. (2017) give a detailed update. They discuss the trailing axial tail of timevariable emission blobs, enclosed by two 'lateral tails' forming a parabola. If the axial tail represents a jet, arguments about its collimation suggest a very low-density surrounding medium, $n_{\mathrm{HI}}<$ $0.007 \mathrm{~cm}^{-3}$.

Giacani et al. (2005) have imaged the region in $\mathrm{H} \mathrm{I}$, and find a void around the pulsar, with a candidate shell $0.4 \mathrm{pc}$ in radius outside the $\mathrm{X}$-ray PWN, comprising about 0.8 solar masses of neutral gas. ${ }^{1}$ This material has a line-of-sight velocity of approximately $+3 \mathrm{~km} \mathrm{~s}^{-1}$ with respect to the local standard of rest, consistent with association

\footnotetext{
${ }^{\star}$ E-mail: greavesJ1@ cardiff.ac.uk

${ }^{1}$ Giacani et al. used an older distance estimate of $160 \mathrm{pc}$, so the size and mass should be increased accordingly.
}

with the pulsar given its close distance. From the radial velocity and proper motion, the space velocity of the pulsar is estimated at $U$, $V, W \sim-10,+35,+210 \mathrm{~km} \mathrm{~s}^{-1}$ (following the method in a righthanded system of Johnson \& Soderblom 1987), i.e. dominated by motion towards the North Galactic Pole. ${ }^{2}$ The total space velocity is lower than the mean for pulsars of approximately $450 \mathrm{~km} \mathrm{~s}^{-1}$ (Lyne \& Lorimer 1994), but is highly supersonic compared to sound speeds in an $\mathrm{HI}$ or $\mathrm{H}$ II medium.

We present here observations of the Geminga region in the midinfrared and submillimetre continuum, suitable for tracing dust. No other submillimetre observations of PWN have been published to our knowledge (although there are studies of dust being newly formed in SNR). The Geminga data are compared here to recent models for shaping of PWN in an inhomogeneous ISM (Yoon \& Heinz 2017) and mass-loading of the nebula through inflow of neutrals (Morlino, Lyutikov \& Vorster 2015). Further, we identify a submillimetre point source at the pulsar position, with a spectral energy distribution (SED) consistent with cold dust. Given the crowded sightlines near the Galactic plane, this may be a positional coincidence (with a proper motion test not possible at our resolution). However, it could represent circum-pulsar material out of which planets could form, a possibility we are following up with higher resolution ALMA observations.

\section{DATA}

A candidate shell structure around Geminga was noticed in the 12$\mu \mathrm{m}$ image of the region obtained by the Wide-field Infrared Survey Explorer (WISE) satellite. The WISE mapped the entire sky through

\footnotetext{
${ }^{2}$ The parallax uncertainty $(\pi=4.0 \pm 1.3$ mas) allows the out-of-plane motion to lie between about +160 and $+310 \mathrm{~km} \mathrm{~s}^{-1}$.
} 
2010, with a 40-cm telescope operating at wavelengths of 3.4, 4.6, 12 and $22 \mu \mathrm{ms}$. We used the AllWISE data release of 2013, with pixel scales of 1.375 arcsec. In the $12 \mu \mathrm{m}$ band (W3), the full-width at half-maximum (FWHM) of the point spread function is 6.5 arcsec. The left-hand side of the WISE frame may be affected by a roughly vertical stripe apparent on larger scales in the downloaded data. The range of signals shown in Fig. 1 corresponds to approximately $6.5 \sigma$. The signals were left in DN/pixel as the conversion to flux units is not well characterized for cool blackbodies in the broad W3 filter, and it is also difficult to perform aperture photometry in this crowded frame. The two shorter bands $(W 1, W 2)$ are dominated by point sources, and the longer (W4) band is very confused. Danilenko et al. (2011) discuss IR fluxes from Spitzer observations; the W3 band of WISE falls in a gap of Spitzer wavelength coverage (Fig. 3).

We observed the Geminga region twice using Director's time on the 15-m James Clerk Maxwell Telescope (JCMT) in Hawaii. The diffraction limited resolution of the JCMT is $8 \operatorname{arcsec}$ at $450 \mu \mathrm{m}$ and 14 arcsec at $850 \mu \mathrm{m}$, with both wavebands observed simultaneously. The original search in 1999 (January 13 to February 14) with the SCUBA camera was motivated by the announcement of a planet candidate, in pulse timing measurements that were later attributed to timing noise (Mattox, Halpern \& Caraveo 1999). The SCUBA upper limits were not published at the time, but the data were reprocessed later with more mature techniques. In particular, an unevenly sampled image spanning 2 arcmin could be made from the $0.45-\mathrm{mm}$ data, even though a photometry observing mode had been used. The bolometers were spaced two beams apart on the sky, but the gaps were partially filled by sky rotation and the $3 \times$ 3 2-arcsec-spaced jiggle pattern used to compensate for pointing drifts. The data were calibrated using sky opacity measurements at the time of observation and standard flux conversion factors.

This $0.45-\mathrm{mm}$ image made retrospectively suggested emission both towards the pulsar and in the nebula, so a second award of Director's time was used for follow-up, in 2013 (October 5 to November 26) with the SCUBA-2 camera. This field of view covered about 10 arcmin, in a daisy-scan map mode. The data reduction was tuned to extract compact features, by zero masking, so any low surface brightness extended structure tends to be removed. Standard reduction procedures were used; the flux conversion factor during the observations was about 3 per cent below the nominal value. A blank region identified ahead of the pulsar's track (top left-hand side of the frame in Fig. 1) was used to establish a nominal zero level for the SCUBA image (adding a constant of $15 \mathrm{mJy}^{\text {beam }}{ }^{-1}$ ).

The SCUBA-2 $0.85-\mathrm{mm}$ data set is dominated by diffuse structures of low signal-to-noise, including a patch of emission around Geminga. The SCUBA $0.85 \mathrm{~mm}$ photometry could not be reconstructed into a map as the bolometers are spaced further apart than at $0.45 \mathrm{~mm}$, but a flux-density measurement was made towards the pulsar (Table 1). Apart from this measurement, all the SCUBA and SCUBA-2 maps data sets been smoothed by Gaussians of 7-8 arcsec FWHM, to improve the image clarity.

\section{RESULTS}

\subsection{Shell structure}

The WISE 12-micron image suggests a shell-like parabolic structure comprising a number of clumps (with some possible $0.45 \mathrm{~mm}$ counterparts). This shell is reminiscent of the infrared loop around the young SNR G54.1+0.3, discovered with Akari at $15 \mu \mathrm{m}$ (Koo et al. 2008). Infrared emission can come from warm dust and/or gas (e.g. Temin 2009) when the ambient medium is compressed. The
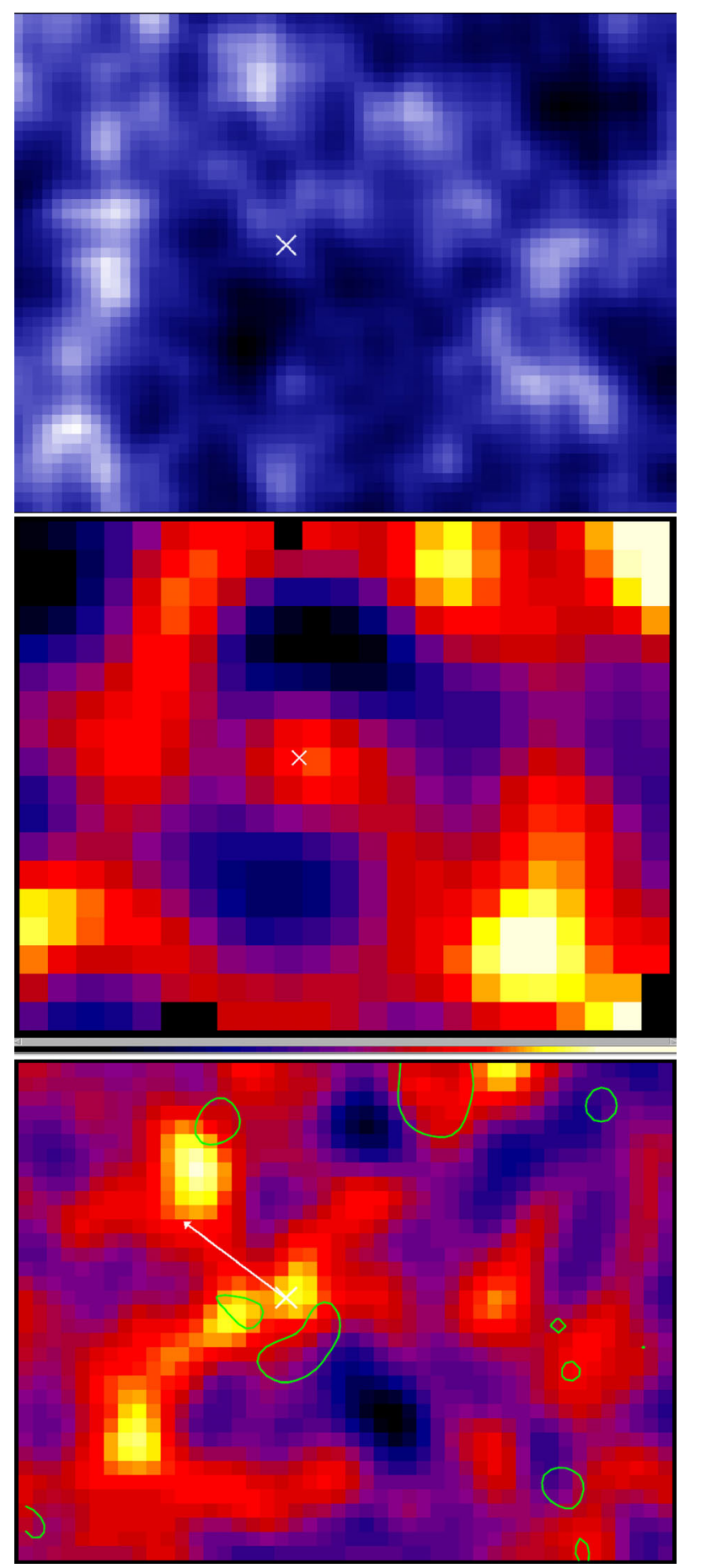

Figure 1. Fields around Geminga: each 90 by 70 arcsec in RA, Dec., corresponding to the region in the X-ray image in Fig. 2. The white crosses indicate the J2000 pulsar position of RA, Dec. $=06: 33: 54.153,+17: 46: 12.91$. Top panel: WISE $12 \mu \mathrm{m}$ image, with linear intensity scale from 811.7 to 819.4 DN/pixel, and uncertainties of $\approx 1.2 \mathrm{DN} /$ pixel. Middle panel: epoch 1999.1 SCUBA image at $0.45 \mathrm{~mm}$, with a $40 \mathrm{mJy}^{\text {beam }}{ }^{-1}$ flux-density (90 per cent) range, on a linear colour scale (shown underneath); the holes (black pixels) are due to uneven sampling (see text). Bottom panel: epoch 2013.8 SCUBA-2 image at $0.45 \mathrm{~mm}$, using the same $40 \mathrm{mJy}$ beam $^{-1}$ colour-scale (here 84 per cent of the signal range). The green contour is $2 \mathrm{mJy} \mathrm{beam}^{-1}(2 \sigma)$ in the simultaneous $0.85 \mathrm{~mm}$ data. The white arrow shows the proper motion; in 2013, the pulsar was located 2 arcsec up this arrow compared to the $\mathrm{J} 2000$ position (white cross). 
Table 1. Peak flux-densities in JCMT data, in mJy beam ${ }^{-1}$. S and S-2 denote observations with SCUBA and SCUBA-2, respectively. The ahead and behind directions refer to the pulsar's motion, e.g. ahead is roughly NE. The $0.85 \mathrm{~mm}$ SCUBA-2 peaks are offset by several arcsec from those at $0.45 \mathrm{~mm}$, which can be an artefact at low signal-to-noise ratios. The $0.85 \mathrm{~mm}$ SCUBA photometry gave a flux-density of $2.5 \pm 1.1 \mathrm{mJy}^{\text {beam }}{ }^{-1}$ towards the pulsar.

\begin{tabular}{lccc}
\hline location & $0.45 \mathrm{~mm}(\mathrm{~S}-2)$ & $0.45 \mathrm{~mm}(\mathrm{~S})$ & $0.85 \mathrm{~mm}(\mathrm{~S}-2)$ \\
\hline Geminga & $29 \pm 9$ & $27 \pm 8$ & $2.5 \pm 1$ \\
$20-25$ arcsec ahead & $36 \pm 9$ & $28 \pm 8$ & $3.5 \pm 1$ \\
$47-45$ arcsec behind & $20 \pm 9$ & $36 \pm 8$ & $4 \pm 1$ \\
\hline
\end{tabular}

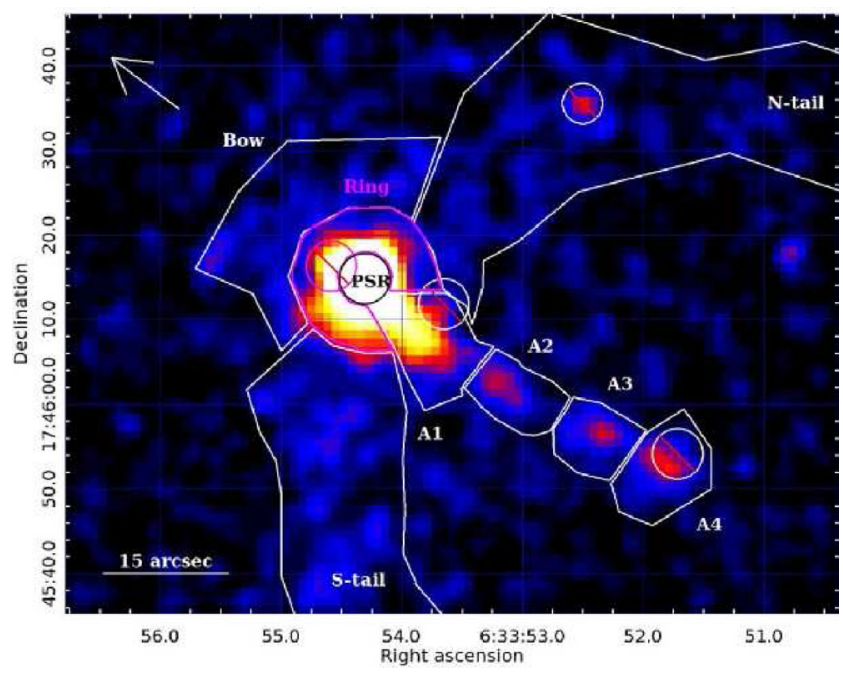

Figure 2. Chandra $0.3-7 \mathrm{keV}$ image of the Geminga region, matching the Fig. 1 fields (reproduced by permission of the AAS from fig. 1 of 'Geminga's Puzzling Pulsar Wind Nebula', by B. Posselt, G. G. Pavlov, P. O. Slane, R. Romani, N. Bucciantini, A. M. Bykov, O. Kargaltsev, M. C. Weisskopf and C.-Y. Ng, in The Astrophysical Journal, Vol 835, Issue 1, 2017, article id 66). The pulsar position is labelled PSR and the arrow shows the direction of motion. The $\mathrm{N}$ and $\mathrm{S}$ lateral tails and the axial (A) tail are outlined. The outlines mark regions used for signal-extraction, with summed X-ray counts of $\approx 0.35$ per cent -15 per cent of the peak signal within the labelled areas. The crossed circles contain unrelated point sources.

signal-to-noise ratio of the WISE image is modest and the line of sight will be crowded only a few degrees above the Galactic plane, so deeper observations would help to confirm that this is a coherent structure. The structure does not appear to be made from chance groupings of stars, from inspection of the 3.4 and $4.6 \mu \mathrm{m}$ images from WISE and the $5.8 \mu \mathrm{m}$ Spitzer image constructed by Danilenko et al. (2011).

The two X-ray lateral tails ( $\mathrm{N}$ and $\mathrm{S}$ in Fig. 2 ) overlay the faintest parts of the $12 \mu \mathrm{m}$ signal (Fig. 1). The mechanism for producing the $\mathrm{X}$-rays is unclear (Posselt et al. 2017), but the image comparison suggests the X-ray source regions lie in cavities in the IR-emitting material.

The infrared loop appears tilted with respect to the direction of pulsar motion. This effect has been simulated recently by Yoon \& Heinz (2017), in models where a PWN passes through an inhomogeneous ISM, including motion at an angle through a density gradient. The model addressed the case of PSR J2124-3358, where the axis of the nebula is observed to be offset by $\sim 20^{\circ}$ compared to the direction of motion. If Geminga is slowly passing to lower densities as it moves away from the Galactic plane, a similar misalignment could occur.

\subsection{Forwards and reverse features}

Candidate bow-front emission is seen at $0.45 \mathrm{~mm}$, in both the SCUBA and SCUBA-2 images, lying approximately 20-25 arcsec ahead of the pulsar. In the WISE $12 \mu \mathrm{m}$ image, the front of the parabola of emission is approximately $30 \mathrm{arcsec}$ ahead of Geminga. The stand-off radius where the PWN meets the ISM is expected to be at

$R_{\mathrm{s}}=\left(\dot{E}_{\text {wind }} f / 4 \pi c \rho_{0} v_{*}^{2}\right)^{1 / 2}$,

where for space motion $v_{*}$ of $210 \mathrm{~km} \mathrm{~s}^{-1}$ and an isotropic $(f=$ 1) wind in a medium with number density $n_{0}<0.007 \mathrm{H} \mathrm{I} \mathrm{cm}^{-3}$, Posselt et al. (2017) find $R_{\mathrm{s}}>34 \operatorname{arcsec} \sqrt{\xi}$, where $\xi=\dot{E}_{\text {wind }} / \dot{E}$ is the fraction of the power emitted in the wind. For a stand-off point at $25 \pm 5$ arcsec ahead of the pulsar, $\xi \lesssim 0.5$ (range of $\sim 0.35-0.8$, including the uncertainty in offset but neglecting that in space velocity). The power-fraction is poorly constrained but the calculation does demonstrate that this is a reasonable location for the bow-shock candidate, given a low ISM density around Geminga.

A further $0.45-\mathrm{mm}$ flux peak is seen behind the pulsar, at the end of the axial tail seen in X-rays (near A4 in Fig. 2). The distance from Geminga is approximately $37-45$ arcsec, with SCUBA and SCUBA-2 locations differing by about one beam, and the signals by $1.3 \sigma$ (combining the noise measurements in quadrature). The time sampling and resolution are too poor to test for moving clumps, as was done for the X-ray tail features (Posselt et al. 2017).

There are weak peaks in the 0.85 -mm data, both ahead and behind the pulsar. The offsets compared to the $0.45 \mathrm{~mm}$ peaks (Fig. 1) may be artefacts of low signal-to-noise ratio in large beams (e.g. Holland et al. 2017).

\subsection{Pulsar location}

Table 1 lists the flux-densities in the beams towards Geminga. The 0.45 -mm source is compact in each image, while there is slightly extended $0.85-\mathrm{mm}$ flux around the pulsar in the 2013 image. From the peak flux-densities, the $0.45-0.85 \mathrm{~mm}$ spectral index is $\approx 3.8 \pm$ 0.6 , or higher if the $0.85 \mathrm{~mm}$ flux is contaminated by other lineof-sight sources. For a spectral index $\alpha$ (flux rising with frequency according to $F_{v} \propto v^{\alpha}$ ), the value for Geminga is consistent with dust emission. In the Rayleigh-Jeans regime, grains with emissivity proportional to $\nu^{\beta}$ yield $\alpha=\beta+2$, so here $\beta$ is $\gtrsim 1.2$. This suggests grains somewhat smaller in size than the wavelengths of observation (if they are comparable, $\beta$ tends to zero), and allows particles as small as ISM grains $(\beta \approx 1.8)$.

Fig. 3 shows the SED towards Geminga, combining the submillimetre detections with other data. An example blackbody spectrum is superposed; the $30 \mathrm{~K}$ dust temperature fits the four submillimetre points and the Spitzer $24 \mu \mathrm{m}$ upper limit (Danilenko et al. 2011). A modified greybody fit (accounting for $\beta>0$ ) would improve the fit to the $0.85-\mathrm{mm}$ measurements. There is a substantial submillimetre excess over extrapolations of the optical or radio flux, and no other mechanism applicable to this waveband that could produce the steep submillimetre spectral index. A dust temperature of around $30 \mathrm{~K}$ is comparable to the mid-plane temperatures in protoplanetary discs. 


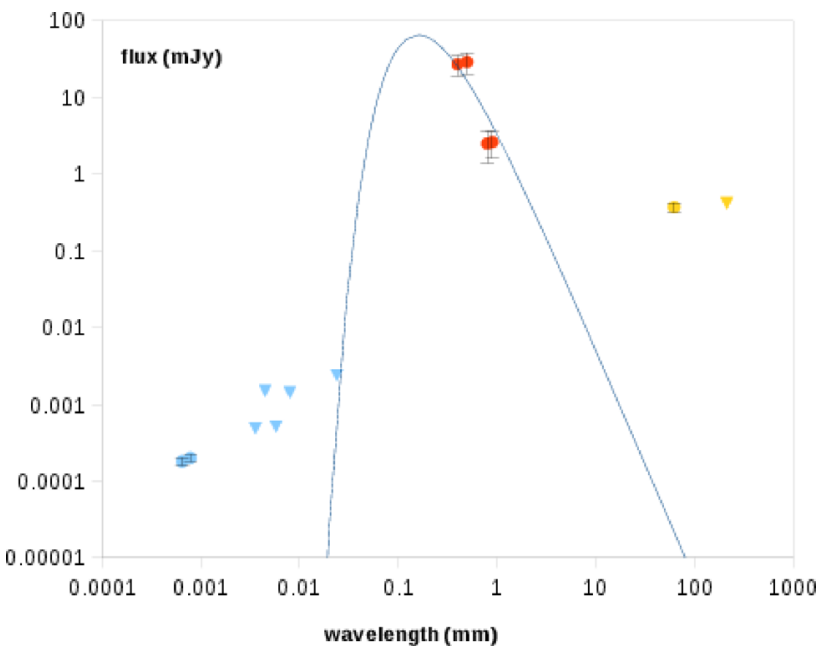

Figure 3. SED of signals measured towards Geminga. The SCUBA and SCUBA-2 results are shown slightly offset horizontally, for clarity. The blue curve is an empirical $30 \mathrm{~K}$ dust fit (see text). The $R, I$-band de-reddened signals (blue circles) are from Shibanov et al. (2006); the Spitzer upper limits (blue arrowheads) at 3.6, 4.5, 5.8, 8, $24 \mu \mathrm{m}$ are from Danilenko et al. (2011) and the radio points (yellow symbols) are at $6.3 \mathrm{~cm}$ (Pellizzoni et al. 2011) and $21 \mathrm{~cm}$ (Giacani et al. 2005). The $3.6 \mu \mathrm{m}$ point is a possible detection, with a large uncertainty; the $6.3 \mathrm{~cm}$ feature is slightly extended and probably part of the axial tail (Pellizzoni et al. 2011).

\section{DISCUSSION}

We consider whether ISM dust that interacts with the PWN could penetrate inside the nebula. This 'mass loading' has recently been modelled by Morlino et al. (2015), although in the context of neutral hydrogen flowing inwards and affecting the pulsar tail. For Geminga, the $\mathrm{H}_{\mathrm{I}}$ shell with a radius of 9 arcmin (Giacani et al. 2005) suggests a suitable reservoir of hydrogen outside the PWN; such parsec-scale structure could be associated with a pulsar-driven bubble (Yoon \& Heinz 2017).

Morlino et al. (2015) show that for neutron star velocities under about $300 \mathrm{~km} \mathrm{~s}^{-1}$, neutral atoms can penetrate the nebula without interacting with protons in the shocked gas. The interaction length needs to exceed $(5 / 16) R_{\mathrm{s}}$, where $R_{\mathrm{S}} \approx 25 \operatorname{arcsec}$ corresponds to $9 \times$ $10^{14} \mathrm{~m}$. The interaction length can be written as

$\lambda=v_{*} /\left[X_{\text {ion }} n_{\mathrm{ISM}} r_{\mathrm{c}} \sigma v_{\mathrm{p}}\right]$,

with constants for the ionization fraction $X_{\text {ion }}$ and shock compression ratio $r_{\mathrm{c}}$ set to approximately 0.9 and 4, respectively (Morlino et al. 2015). The velocity $v_{\mathrm{p}}$ of the protons can be approximated to $\sqrt{16 k T / \pi m_{\mathrm{p}}}$ or $15 \mathrm{~km} \mathrm{~s}^{-1}$ at temperatures $T$ of around $15000 \mathrm{~K}$ (inferred from the $\approx 1-1.5 \mathrm{keV}$ peaks of SEDs for regions ahead of the pulsar, Posselt et al. 2017). Then for the upper limit on the density $n_{\mathrm{ISM}}=0.007 \mathrm{~cm}^{-3}, \lambda=3.3 \times 10^{8} / \sigma$. If penetration requires $\lambda>3 \times 10^{14} \mathrm{~m}$ (i.e. $5 / 16 R_{\mathrm{s}}$ ), the particle interaction cross-section should be smaller than $1.2 \times 10^{-6} \mathrm{~m}^{2}$. If we can set this to be the physical size of a dust particle, its dimensions must be under about $1 \mathrm{~mm}$.

Thus the calculation suggests that grains could penetrate the PWN without interacting within the shocked zone. This size of dust particle is consistent with the spectral index found above for the point source, and it is also similar to dust-aggregates of mm-sizes found in protoplanetary discs.
Analytical calculations for circumpulsar dust (Löhmer, Wolszczan \& Wielebinski 2004; Foster \& Fischer 1996; Greaves \& Holland 2000; Koch-Miramond et al. 2002) have assumed a disc similar to that of a young star, with grains heated by intercepting a fraction $f^{\prime}$ of the spin-down luminosity of the pulsar. In a model with a spectral peak around $100 \mu \mathrm{m}$ (as in our Fig. 3 fit), Löhmer et al. (2004) show that 1 Earth-mass of dust at 250 pc would emit up to $0.1 \mathrm{mJy}$ at $0.45 \mathrm{~mm}$ for $f^{\prime}=0.01$, if the grains have sizes within the range of $0.01 \mu \mathrm{m}-1 \mathrm{~mm}$. Adopting $f^{\prime} \lesssim 0.5$ (allowing up to $\xi$ $\sim 0.5$ of power in the wind, although this was poorly constrained), we can convert our point-source $0.45-\mathrm{mm}$ flux of $\sim 30 \mathrm{mJy}$ to a minimum of about 6 Earth-masses of dust.

\section{CONCLUSIONS}

The origins of the rare pulsar planet systems are uncertain, with recent work (Margalit \& Metzger 2017) favouring disruption of a companion over re-accretion of supernova fallback material. Here, we find evidence that the middle-aged ${ }^{3}$ Geminga pulsar is surrounded by a shell of material that could have formed from compression of the local ISM. Preliminary calculations suggest that dust could penetrate the nebula, given the low space speed and local density, and this may provide an alternate source for dust near this pulsar. A candidate circum-pulsar disc would be the first to be found in the submillimetre, complementing the only infrared candidate (around the magnetar 4U 0142+61, Wang, Chakrabarty \& Kaplan 2006). We are waiting for higher resolution follow-up data, but can infer that any dust disc present around Geminga should exceed about 6 Earth-masses of dust. Thus, it would have potential to form low-mass planets, such as the archetypes around PSR B1257+12 (Wolszczan \& Frail 1992).

\section{ACKNOWLEDGEMENTS}

We thank the former Directors of the JCMT, Ian Robson and Gary Davis, for their generous awards of discretionary time.

\section{REFERENCES}

Danilenko A. A., Zyuzin D. A., Shibanov Yu. A., Zharikov S. V., 2011, MNRAS, 415, 867

Faherty J., Walter F. M., Anderson J., 2007, Ap\&SS, 308, 225

Foster R. S., Fischer J., 1996, ApJ, 460, 902

Giacani E., Reynoso E. M., Dubner G., Goss W. M., Green A. J., Johnston S., 2005, Adv. Space Res., 35, 1070

Greaves J. S., Holland W. S., 2000, MNRAS, 316, L21

Holland W. S. et al., 2017, MNRAS, preprint (arXiv:1706.01218)

Johnson D. R. H., Soderblom D. R., 1987, AJ, 93, 864

Koch-Miramond L., Haas M., Pantin E., Podsiadlowski Ph., Naylor T., Sauvage M., 2002, A\&A, 387, 233

Koo B. -C. et al., 2008, ApJ, 673, L147

Linden T., Auchettl K., Bramante J., Cholis I., Fang K., Hooper D., Karwal T. Weishi Li S., 2017, preprint (arXiv:1703.09704)

Löhmer O., Wolszczan A., Wielebinski R., 2004, A\&A, 425, 763

Lyne A. G., Lorimer D. R., 1994, Nature, 369, 127

Margalit B., Metzger B. D., 2017, MNRAS, 465, 2790

Mattox J. R., Halpern J. P., Caraveo P. A., 1999, BAAS, 31, 904

${ }^{3}$ Middle-aged is defined as, for example, 100-400 kyr old by Linden et al. (2017). 
Morlino G., Lyutikov M., Vorster M., 2015, MNRAS, 454, 3886

Pellizzoni A., Govoni F., Esposito P., Murgia M., Possenti A., 2011, MNRAS, 416, L45

Posselt B. et al., 2017, ApJ, 835, 66

Shearer A. et al., 1998, A\&A, 355, L21

Shibanov Y. A. et al., 2006, A\&A, 448, 313

Temin T., 2009, PhD thesis, Univ. Minnesota
Wang Z., Chakrabarty D., Kaplan D. L., 2006, Nature, 440, 772

Wolszczan A., Frail D., 1992, Nature, 355, 145

Yoon D., Heinz S., 2017, MNRAS, 464, 3297

This paper has been typeset from a $\mathrm{T}_{\mathrm{E}} \mathrm{X} / \mathrm{L} \mathrm{T}_{\mathrm{E}} \mathrm{X}$ file prepared by the author. 